\title{
Strong enhancement of phonon scattering through nanoscale grains in lead sulfide thermoelectrics
}

\author{
Haijun $\mathrm{Wu}^{1,2,6}$, Jesús Carrete ${ }^{3,6}$, Zhiyun Zhang ${ }^{2}$, Yongquan $\mathrm{Qu}^{2}$, Xuetao Shen ${ }^{2}$, Zhao Wang ${ }^{2,4}$, Li-Dong Zhao ${ }^{5}$ \\ and Jiaqing $\mathrm{He}^{1}$
}

We present nanocrystalline $\mathrm{PbS}$, which was prepared using a solvothermal method followed by spark plasma sintering, as a promising thermoelectric material. The effects of grains with different length scales on phonon scattering of PbS samples, and therefore on the thermal conductivity of these samples, were studied using transmission electron microscopy and theoretical calculations. We found that a high density of nanoscale grain boundaries dramatically lowered the thermal conductivity by effectively scattering long-wavelength phonons. The thermal conductivity at room temperature was reduced from $2.5 \mathrm{~W} \mathrm{~m}^{-1} \mathrm{~K}^{-1}$ for ingot-PbS (grain size $>200 \mu \mathrm{m}$ ) to $2.3 \mathrm{~W} \mathrm{~m}^{-1} \mathrm{~K}^{-1}$ for micro-PbS (grain size $>0.4 \mu \mathrm{m}$ ); remarkably, thermal conductivity was reduced to $0.85 \mathrm{~W} \mathrm{~m}^{-1} \mathrm{~K}^{-1}$ for nano-PbS (grain size $\sim 30 \mathrm{~nm}$ ). Considering the full phonon spectrum of the material, a theoretical model based on a combination of first-principles calculations and semiempirical phonon scattering rates was proposed to explain this effective enhancement. The results show that the high density of nanoscale grains could cause effective phonon scattering of almost $61 \%$. These findings shed light on developing high-performance thermoelectrics via nanograins at the intermediate temperature range.

NPG Asia Materials (2014) 6, e108; doi:10.1038/am.2014.39; published online 27 June 2014

Keywords: grain; nanoscale; phonon; thermal conductivity; thermoelectrics

\section{INTRODUCTION}

Thermoelectric materials capable of scavenging electric power from sources of waste heat are currently attracting significant attention as part of the search for sustainable alternative energy sources. ${ }^{1,2}$ For thermoelectric applications, the performance of a material is evaluated by its dimensionless figure of merit $Z T=\left(S^{2} \sigma / \kappa\right) T$, where $S$, $\sigma, \kappa$ and $T$ are the Seebeck coefficient, electrical conductivity, thermal conductivity and absolute temperature, respectively. For a material to have excellent thermoelectric performance, a combination of a high power factor $\left(S^{2} \sigma\right)$ and low thermal conductivity $(\kappa)$ is required. ${ }^{1,2}$ However, the interdependence of $S, \sigma$ and $\kappa$ complicates the development of strategies for improving $Z T$ beyond 3.0, a feat that could revolutionize thermal energy conversion efficiency. Thus far, nanostructuring (that is, embedding nanoscale precipitates) in bulk materials has been shown to be a particularly effective route for enhancing $Z T$ by significantly lowering thermal conductivity. ${ }^{3-5}$ However, the existence of nanoscale precipitates also intensifies the scattering of charge carriers that, in turn, decreases the power factor. Furthermore, controlling the size and morphology of nanoscale precipitates embedded in a parent matrix remains challenging for material synthesis. Therefore, alternative methods capable of reducing thermal conductivity as effectively as nanoscale precipitates but with improved control are desirable. Introducing grain boundary scattering in nanocrystalline bulk materials is a promising strategy to achieve this goal. ${ }^{6}$ In contrast to nanoscale precipitates, nanograin phonon scattering centers are an intrinsic material feature that can be well controlled, and it simplifies device fabrication as well as integration into a larger system and potentially prolongs material lifetime.

Grain boundary phonon scattering has been shown to be important for improving the thermoelectric performance of $\mathrm{Bi}_{2} \mathrm{Te}_{3}$, PbTe- and SiGe-based alloys. ${ }^{6-14}$ Notably, Ren and co-workers ${ }^{6,8,12}$ achieved significant enhancements of $Z T$ in nanograined bulk $\mathrm{Bi}_{2} \mathrm{Te}_{3}$-based thermoelectric materials by exploiting nanoscale grain boundary scattering to achieve reduced thermal conductivity.

\footnotetext{
${ }^{1}$ Department of Physics, South University of Science and Technology of China, Shenzhen, People's Republic of China; ${ }^{2}$ Frontier Institute of Science and Technology, Xi'an Jiaotong University, Xi'an, People's Republic of China; ${ }^{3}$ Departamento de Física de la Materia Condensada, Facultad de Física, Universidad de Santiago de Compostela, Santiago de Compostela, Spain; ${ }^{4}$ Center for Advancing Materials Performance from the Nanoscale (CAMP-Nano), State Key Laboratory for Mechanical Behavior of Materials, Xi'an Jiaotong University, Xi'an, People's Republic of China and ${ }^{5}$ Department of Chemistry, Northwestern University, Evanston, IL, USA

${ }^{6}$ These authors contributed equally to this work.

Correspondence: Professor JQ He, Department of Physics, South University of Science and Technology of China, Shenzhen 518055, People's Republic of China.

E-mail: he.jq@sustc.edu.cn

or Dr L-D Zhao, Department of Chemistry, Northwestern University, 2145 Sheridan Road, Evanston, IL 60208, USA.

E-mail: lidong-zhao@northwestern.edu

or Dr YQ Qu, Frontier Institute of Science and Technology, Xi'an Jiaotong University, Xi'an 710054, People's Republic of China.

E-mail: yongquan@mail.xjtu.edu.cn (for the preparation of PbS nanoparticles)
}

Received 10 September 2013; revised 27 March 2014; accepted 14 April 2014 
This success has provided motivation for subsequent research, both experimental and theoretical, on nanograins. ${ }^{7,9-11,13,14}$ Recently, Biswas et al. ${ }^{9}$ reported high-performance bulk thermoelectrics $\mathrm{PbTe}-4 \% \mathrm{SrTe}-2 \% \mathrm{Na}$ with multiscale hierarchical architectures in which mesoscale grain boundaries could effectively scatter phonons with long mean free paths. Thus, it would be interesting to explore nanoscale grain boundaries for lattice thermal conductivity reduction. When a high density of boundaries or interfaces between nanoscale grains is introduced throughout thermoelectric materials, phonons can be intensively and extensively scattered and thermal conductivity is significantly reduced. ${ }^{9}$ It is well known that charge carriers can also be strongly scattered by abundant nanograin boundaries, leading to a pronounced decrease in electrical conductivity. However, a decrease in thermal conductivity resulting from increased phonon scattering at grain boundaries can compensate for diminished carrier mobility ${ }^{15}$ to achieve a net increase in $Z T$ by keeping a balance between thermal transport and electrical mobility.

In the present work, we performed a systematic study of the roles of grains with varied length scales, from micro to nano, with a special emphasis on the thermal transport properties of $\mathrm{PbS}$. The results indicated that nanograins with a high density of grain boundaries are more effective for lowering thermal conductivity than nanoprecipitates and are also effective for suppressing the bipolar effect. A high $Z T$ value of 0.5 was achieved for nanocrystalline $\mathrm{PbS}$ materials at $923 \mathrm{~K}$, almost a twofold enhancement with respect to the 0.3 measured at $923 \mathrm{~K}$ for a grain boundary-free ingot-PbS.

\section{EXPERIMENTAL PROCEDURES}

\section{Starting materials}

Lead (II) oxide, $\mathrm{Pb}$ wire (99.99\%, American Elements, Los Angeles, CA, USA), S shot or chunk (99.999\%, Inc., Canada), 4-Aminothiophenol, oleic acid, diphenyl ether, 1-octadecene and oleylamine (OAm) were purchased from Sigma-Aldrich (St Louis, MO, USA) and used as received without further purification.

\section{Preparation of $\mathrm{PbS}$ nanoparticles}

For a typical synthesis, $4.5 \mathrm{mmol}$ of lead (II) oxide, $21 \mathrm{ml}$ of diphenyl ether and $9 \mathrm{ml}$ of oleic acid were mixed in a three-neck flask. The mixture was heated to $150{ }^{\circ} \mathrm{C}$ under argon $(\mathrm{Ar})$ protection until a clear $\mathrm{Pb}$ source solution was obtained. A sulfur-OAm precursor solution was prepared separately in a threeneck flask by dissolving $3 \mathrm{mmol}$ of sublimed sulfur powder in $3 \mathrm{ml} \mathrm{OAm}$ at $100{ }^{\circ} \mathrm{C}$ for $1 \mathrm{~h}$ under Ar protection. After cooling to room temperature, $4.5 \mathrm{ml}$ of OAm and $22.5 \mathrm{ml}$ of diphenyl ether were added. Subsequently, the sulfur$\mathrm{OAm}$ precursor solution was quickly transferred into the $\mathrm{Pb}$-source solution. To avoid the introduction of air into the reaction system, the transfer process was protected by an $\mathrm{Ar}$ atmosphere. The temperature of the reaction solution was increased and kept at $180^{\circ} \mathrm{C}$ for $10 \mathrm{~min}$ to allow growth of $\mathrm{PbS}$ nanoparticles to the desired size. The reaction was then quenched by immersing the flask in an ice bath. The $\mathrm{PbS}$ nanoparticles were precipitated by ethyl alcohol, centrifuged for three cycles and dried under vacuum. The isolated $\mathrm{PbS}$ nanoparticles were redispersed in hexane to form a stable colloidal suspension suitable for future characterization. Then, 5.0 and $6.0 \mathrm{mmol}$ of $\mathrm{Pb}$ precursors were used for the synthesis of the nano-PbS-2 and nano-PbS-3 samples with 1 at $\%$ and 2 at $\%$ excess $\mathrm{Pb}$, respectively. For a typical ligand exchange reaction, $1.0 \mathrm{~g}$ oleic acid capped $\mathrm{PbS}$ nanoparticles were dispersed in $100 \mathrm{ml}$ of methanol to achieve a typical nanoparticle concentration of $10 \mathrm{mg} \mathrm{ml}^{-1}$. The obtained solution was degassed with Ar for $30 \mathrm{~min}$ to remove any $\mathrm{O}_{2}$. Then, $1.0 \mathrm{~g} 4$-aminothiophenol was added and the mixture was stirred at room temperature for $16 \mathrm{~h}$ under Ar protection. The $\mathrm{PbS}$ nanoparticles were centrifuged and washed with methanol three times. The obtained solid was dried under vacuum and stored inside a nitrogen glove box. The prepared nanoparticles were thoroughly washed by multiple precipitation and redispersion steps. Washed nanoparticles were dried under an $\mathrm{Ar}$ atmosphere. To completely remove any residual organic ligands, the nanoparticles were heated to $500{ }^{\circ} \mathrm{C}$ for $1 \mathrm{~h}$ under an Ar flow inside a tube furnace. The annealed nanoparticles were ground into a fine powder.

Synthesis of $\mathrm{PbS}$ thermoelectric materials with varied grain sizes Ingot- $\mathrm{PbS}$ was prepared by a melting reaction. Briefly, elemental $\mathrm{Pb}$ and $\mathrm{S}$ were mixed inside carbon-coated fused silica tubes. The tubes were then evacuated to a base pressure of $\sim 10^{-4}$ torr, flame-sealed, slowly heated to $723 \mathrm{~K}$ over $12 \mathrm{~h}$, heated to $1423 \mathrm{~K}$ over $7 \mathrm{~h}$, soaked at this temperature for $6 \mathrm{~h}$ and subsequently air-quenched to room temperature. The molten samples were intermittently mixed by rocking to reduce ingot bubbles and ensure homogeneous composition. The tubes were subsequently air-quenched to room temperature. Micro- $\mathrm{PbS}$ was prepared by hand-grinding the melt-grown ingots down to a powder size $<5 \mathrm{~mm}^{3}$ using a mortar and pestle, followed by mechanical grinding with a mortar and pestle to reduce the powder size to $<53 \mu \mathrm{m}^{3}$. The grinding was carried out inside an Ar-filled glove box. The powders were subsequently densified using a spark plasma sintering (SPS) (SPS-211Lx, Dr Sinter, Kanagawa, Japan) system at $823 \mathrm{~K}$ with a hold time of $10 \mathrm{~min}$ in a $\Phi=20 \mathrm{~mm}$ graphite die under an axial compressive stress of $60 \mathrm{MPa}$ in an Ar atmosphere. The nano-PbS polycrystallines were prepared by SPS using PbS nanoparticles synthesized by chemical reactions, as mentioned above. The SPS heating profile was the same as that used for micro-PbS.

\section{Thermoelectric property measurement}

The obtained samples were cut into bars with dimensions of $10 \times 3 \times 2 \mathrm{~mm}$ and were used for simultaneous measurements of the Seebeck coefficient and the electrical conductivity using an Ulvac Riko ZEM-3 (Ulvac, Yokohama, Japan) instrument under a helium atmosphere from room temperature to $923 \mathrm{~K}$. The thermal conductivity was calculated from $\kappa=D \cdot C_{\mathrm{p}} \cdot \rho$, where the thermal diffusivity coefficient $D$ was measured using the laser flash diffusivity method on a Netzsch LFA457 (Netzsch, Selb, Germany) instrument, and the data were analyzed using a Cowan model with pulse correction. $C_{\mathrm{p}}$, the specific heat capacity, was indirectly derived using a standard sample (Pyroceram 9606) in the range of $300-923 \mathrm{~K}$. The density $\rho$ was determined from the sample dimensions and masses and was confirmed by measurement with a gas pycnometer (Micromeritics Accupyc1340, Norcross, GA, USA). Hall coefficients were measured on a home-built system in magnetic fields ranging from 0 to $1.25 \mathrm{~T}$, utilizing a simple four-contact Hall-bar geometry in both negative and positive polarity to eliminate Joule resistive errors.

\section{Electron microscopy and X-ray diffraction}

The transmission electron micrograph (TEM) investigations were carried out on a JEOL 2100F (JEOL, Tokyo, Japan) microscope operated at $200 \mathrm{kV}$. Thin TEM specimens were prepared by conventional methods. The procedures included cutting, grinding, dimpling, polishing and Ar-ion milling on a liquid nitrogen cooling stage. Samples pulverized in an agate mortar were used for powder X-ray diffraction (XRD) analysis. The powder diffraction patterns were obtained using $\mathrm{Cu} \mathrm{K}_{\alpha}(\lambda=1.5418 \AA)$ radiation in a reflection geometry on an Inel diffractometer (Inel, Stratham, NH, USA) equipped with a positionsensitive detector and operating at $40 \mathrm{kV}$ and $20 \mathrm{~mA}$.

\section{Theoretical calculation procedure}

A single-unit-cell calculation based on a $11 \times 11 \times 11$ Monkhorst-Pack k-point grid was initially used to determine the equilibrium lattice parameter of the $\mathrm{PbS}$ rock-salt structure. The ab initio result, $0.585 \mathrm{~nm}$, compared favorably with the experimental value of $0.593 \mathrm{~nm}$. Next, the Born effective charges and dielectric tensor of the system were calculated. Finally, we harnessed the symmetry of the unit cell to determine a minimal set of atomic displacements, from which the full interatomic force constant matrix for a $5 \times 5 \times 5$ unit cell could be obtained, and performed the corresponding calculations using a $3 \times 3 \times 3$ Monkhorst-Pack grid. Combining the interatomic force constant matrix, the Born effective charges and the dielectric tensor were obtained, and the full phonon dispersions over the whole Brillion zone were built. Again, comparison with previous theoretical calculations and experimental data yielded reasonable agreements. 


\section{RESULTS AND DISCUSSION}

$\mathrm{PbS}$ nanoparticles

The synthesized $\mathrm{PbS}$ nanoparticles were studied by powder XRD, and the results are shown in Figure 1a. The well-defined sharp peaks in the XRD pattern reveal the high crystallinity of the cubic rock-salt structured PbS nanoparticles (PDF\#78-1055). ${ }^{16}$ Low- and intermediate-magnification TEM images of the $\mathrm{PbS}$ nanoparticles, shown in Figure 1b, indicate a narrow size distribution $(6-12 \mathrm{~nm}$, with $\sim 9 \mathrm{~nm}$ on average) of the PbS nanoparticles with a nearly cubic shape. The selected area electron diffraction (SAED) pattern (Figure 1c), with an aperture including a batch of nanoparticles and carbon background, further confirmed the presence of cubically structured PbS nanoparticles. Furthermore, the high-resolution TEM (HRTEM) image of a typical single particle in Figure 1d clearly shows the cubic lattice fringes of the PbS crystal. The fast Fourier transform (FFT) image along the [001] zone axis, included in the inset of
Figure 1d, further confirmed the cubic structure of the as-synthesized $\mathrm{PbS}$ nanoparticles. The lattice parameter of the $\mathrm{PbS}$ nanoparticles was measured to be $\sim 0.593 \mathrm{~nm}$ that agrees well with the previously reported value of $0.594 \mathrm{~nm} .{ }^{16}$ The high-angle annular dark-field (HAADF) image of a single PbS nanoparticle and its energydispersion X-ray spectroscopy (EDS) spectrum images shown in Figure 1e indicate that the nanoparticles had a uniform distribution of $\mathrm{Pb}$ and $\mathrm{S}$.

\section{Thermoelectric properties}

Three typical nanocrystalline $\mathrm{PbS}$ samples, nano-PbS-1, nano-PbS-2 and nano-PbS-3, were prepared by a SPS method, where nano-PbS-1 is stoichiometric and the amount of excess $\mathrm{Pb}$ in nano-PbS-2 and nano-PbS-3 was $\sim 1$ at.\% and 2 at.\% by EDS, respectively. To make comparisons, 'ingot-PbS' and 'micro-PbS' polycrystalline samples were prepared by a melting reaction as controls; 'micro-PbS' was a
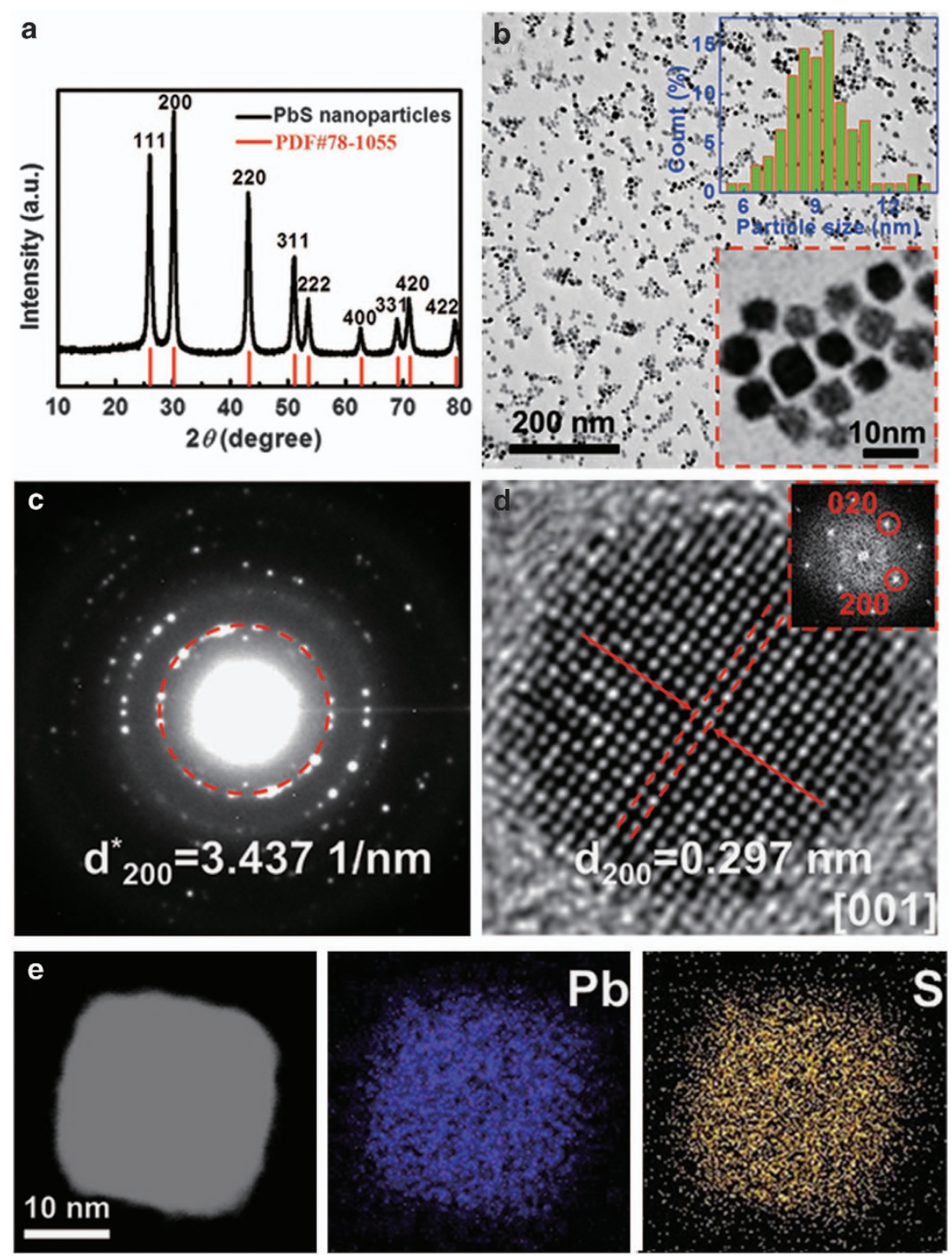

Figure 1 (a) XRD spectrum of the PbS nanoparticles produced. As a reference, the pattern corresponding to the cubic rock-salt PbS phase (PDF\#78-1055) is also shown. (b) TEM image of PbS nanoparticles. The inset shows a histogram of measured particle size. (c) SAED pattern obtained from a batch of nanoparticles and some carbon background. The diameter of the first reflection 200 rings is $\sim 3.4711 \mathrm{~nm}$. (d) HRTEM image of a single nanoparticle with an inserted fast Fourier transform (FFT) image along the [100] zone axis. The 200 fringe is $0.297 \mathrm{~nm}$. (e) HAADF image of a single nanoparticle and its EDS $\mathrm{Pb}$ and $\mathrm{S}$ elemental mappings. 
obtained by pulverization of the ingot in an agate mortar, followed by SPS. The XRD patterns of the ingot-PbS and nano- $\mathrm{PbS}$ samples shown in Supplementary Figure S1 resemble that of the PbS cubic structure without any secondary phases. The full widths at half maximum of the XRD peaks of nano-PbS are wider than those of ingot-PbS, consistent with the smaller particle size of the nano-PbS.

The thermoelectric properties of all the studied $\mathrm{PbS}$ samples, including ingot-, micro- and nano- $\mathrm{PbS}$, are shown in Figure 2. It can be readily observed that the electrical conductivities for ingot- and micro-PbS samples decreased with increasing temperature, indicating a metallic transport behavior (Figure 2a). On the other hand, for the nano-PbS samples the electrical conductivities increased with increasing temperature, similar to what was found in nanocrystalline $\mathrm{PbTe},{ }^{7,16} \mathrm{PbTe}^{-} \mathrm{PbSe}^{17}$ and PbTe-PbS materials. ${ }^{11}$ When the temperature reached $923 \mathrm{~K}$, the electrical conductivities for the three nano-PbS samples were comparable to those of ingot- and micro$\mathrm{PbS}$. The reduced electrical conductivities of nano- $\mathrm{PbS}$ materials at low temperatures could be attributed to the diminished carrier mobility that results from electron scattering at the large number of grain boundaries. ${ }^{7,11,16,17}$ The Hall data show that the carrier mobilities were $\sim 405$ and $277 \mathrm{~cm}^{2} \mathrm{~V}^{-1} \mathrm{~s}^{-1}$ for ingot- and micro$\mathrm{PbS}$, respectively, whereas the carrier mobilities of the nano- $\mathrm{PbS}$ samples were dramatically decreased to $10 \mathrm{~cm}^{2} \mathrm{~V}^{-1} \mathrm{~s}^{-1}$.

Figure $2 \mathrm{~b}$ shows negative values of -205 and $-243 \mu \mathrm{VK}^{-1}$ for ingot-PbS and micro-PbS samples, respectively, at room temperature. In contrast, a very large, positive Seebeck coefficient $\left(609 \mu \mathrm{V} \mathrm{K}^{-1}\right)$ was observed for three nano-PbS samples at room temperature. The Seebeck coefficient for nano-PbS samples decreased with increasing temperature from room temperature to $500 \mathrm{~K}$, and then became negative when the temperature was over $500 \mathrm{~K}$. This transport behavior for the Seebeck coefficient can be associated with a progressive compensation by the thermally excited minor carriers that eventually dominate and cause a change in the sign of the Seebeck coefficient. The Seebeck coefficient can be considered macroscopically as the electrical response of a conductor under the external temperature field, including both the electron and hole contributions. $^{7,17,18}$ If the contributions of electrons and holes to the electrical conductivity are represented by $\sigma_{e}$ and $\sigma_{h}$, respectively, the total Seebeck coefficient $S$ is given by ${ }^{7,17-19}$ :

$$
S=\frac{\sigma_{h}\left|S_{h}\right|-\sigma_{e}\left|S_{e}\right|}{\sigma_{h}+\sigma_{e}}
$$

where $\left|S_{\mathrm{h}}\right|$ and $\left|S_{\mathrm{e}}\right|$ represent the contributions from holes and electrons to the Seebeck coefficient, respectively. Thus, the sign of the total Seebeck coefficient is not a simple comparison between the numbers of electrons and holes, but is instead determined by the values of $\sigma_{\mathrm{e}}\left|S_{\mathrm{e}}\right|$ and $\sigma_{\mathrm{h}}\left|S_{\mathrm{h}}\right|$. In other words, a conductor with a majority of negative carriers can have a positive Seebeck coefficient if the value of $\sigma_{\mathrm{e}}\left|S_{\mathrm{e}}\right|$ is smaller than that of $\sigma_{\mathrm{h}}\left|S_{\mathrm{h}}\right|$. The Hall coefficient measurements indicated that the major charge carriers for $\mathrm{PbS}$ are electrons. Therefore, the positive Seebeck coefficients measured for the nano-PbS samples could be explained by $\sigma_{\mathrm{h}}$ being much higher than $\sigma_{e}$ from room temperature to $\sim 500 \mathrm{~K}$. Because this phenomenon was only observed for the nano-PbS samples, it can likely be explained mechanistically by potential barrier scattering. Briefly, when the grain size is small enough, a potential barrier is formed at the grain boundary. Carriers with higher energies (mostly holes) can easily jump over the potential barrier, whereas lower-energy carriers (mostly electrons) will be filtered out or strongly
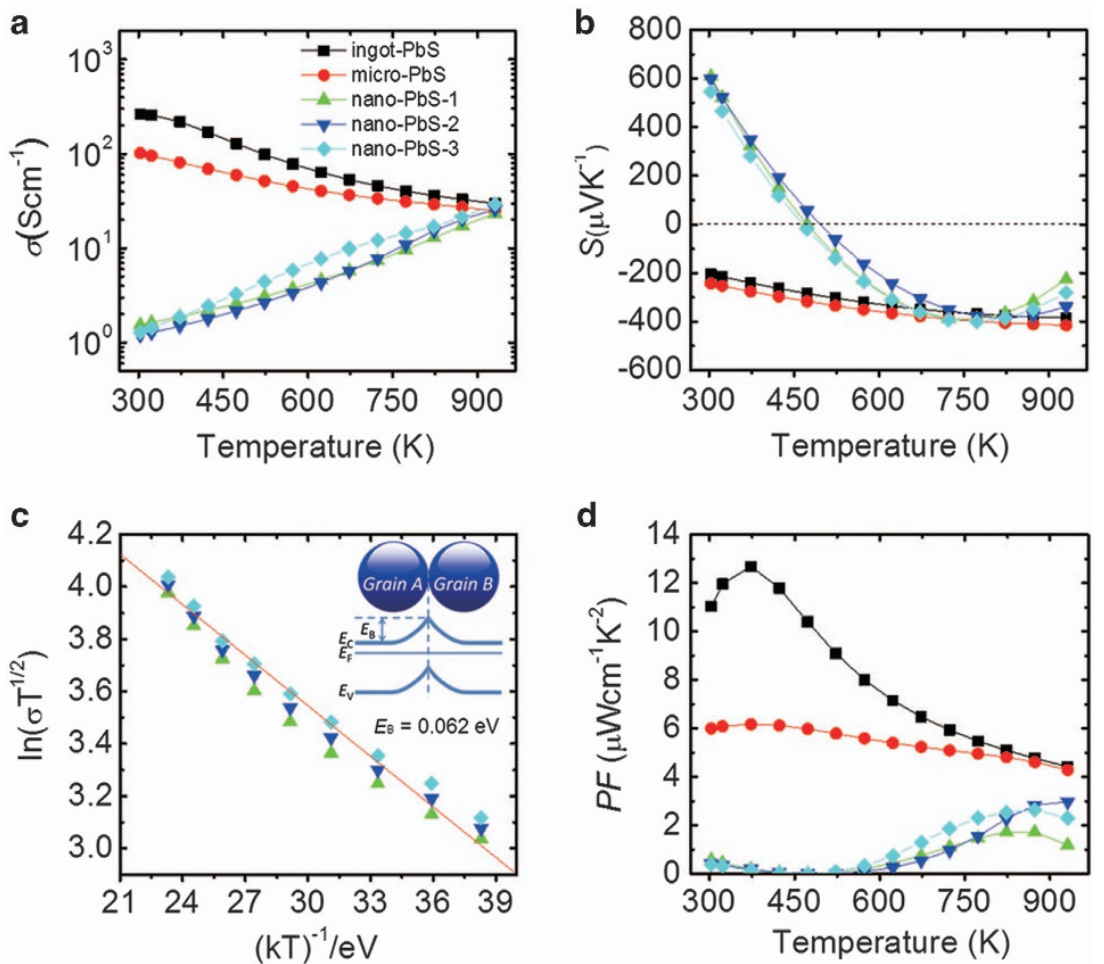

Figure 2 Thermoelectric properties as a function of temperature for PbS samples. (a) Electrical conductivity. (b) Seebeck coefficient. (c) Plot of In( $\sigma T^{1 / 2}$ ) vs $1 /(k T)$ for nano-PbS samples, along with a fit to Equation (2) to derive the energy barrier associated with grain boundary potential barrier scattering. The inset shows the electronic structure at the edges between two grains. (d) Power factor. 
scattered. To estimate the height of the energy barrier at the grain boundaries $\left(E_{\mathrm{B}}\right)$, it can be assumed that the effective electrical conductivity varies according to:

$$
\sigma \propto T^{-1 / 2} \exp \left[\frac{-E_{B}}{k T}\right]
$$

where $k$ is the Boltzmann constant and $T$ is the absolute temperature..$^{20}$ To support this assumption, a plot of $\ln \left(\sigma T^{1 / 2}\right)$ vs $1 /(k T)$ is shown in Figure $2 c$, along with a fit to the above equation that yields a barrier height $E_{\mathrm{B}}$ of $0.062 \mathrm{eV}$. The electronic structure at the boundary between two grains is depicted in the inset as well. This energy barrier can effectively filter out the lower-energy electrons, but fails to stop holes. Thus, the number of holes will be more than that of electrons, resulting in a positive Seebeck coefficient at low temperature. $^{21}$ At elevated temperatures, an increasing number of electrons will gain enough thermal activation energy to jump over the potential barriers at the grain boundaries, eventually leading to the domination of electrons in electrical transport. This scenario explains the negative Seebeck coefficients observed at elevated temperatures. Interestingly, there is an upturn for the Seebeck coefficients of nano$\mathrm{PbS}$ at high temperature. However, there may be complex reasons for this trend. The change in sign of the Seebeck coefficients of the nano$\mathrm{PbS}$ samples indicated that holes and electrons are two competitive carriers in quantity. In addition, the carrier concentration ranges from $1 \times 10^{18}$ to $2 \times 10^{18} \mathrm{~cm}^{-3}$ for nano-PbS that makes the Seebeck coefficient very sensitive to slight changes in charge carrier behavior, especially in the transition from a degenerate to a nondegenerate semiconductor. Furthermore, being at the nanoscale makes the electrical transport properties much more complicated, especially at high temperatures because of factors such as surface-active nanoparticles and changes in the band gap because of nanostructuring. ${ }^{22}$

Figure $2 \mathrm{~d}$ shows the calculated power factor. The power factors of ingot-PbS and micro-PbS showed maximum values of 12.7 and $6.2 \mu \mathrm{W} \mathrm{cm}{ }^{-1} \mathrm{~K}^{-2}$, respectively. The maximum power factor peaks were at higher temperatures for nano-PbS compared with ingot-PbS and micro-PbS. The power factor of nano- $\mathrm{PbS}-2$ peaked at $3 \mu \mathrm{W} \mathrm{cm} \mathrm{cm}^{-1} \mathrm{~K}^{-2}$ at $923 \mathrm{~K}$, nearly two times higher than that of nano-PbS-1 $\left(1.8 \mu \mathrm{W} \mathrm{cm}{ }^{-1} \mathrm{~K}^{-2}\right.$ at $\left.850 \mathrm{~K}\right)$ and slightly higher than that of nano-PbS-3 $\left(2.7 \mu \mathrm{W} \mathrm{cm}{ }^{-1} \mathrm{~K}^{-2}\right.$ at $\left.900 \mathrm{~K}\right)$. The higher power factor of the nano-PbS-2 sample reflects an optimization of the carrier concentration by self-doping with excess $\mathrm{Pb}$.

The total thermal conductivities as a function of temperature for the different samples are shown in Figure 3a. The heat capacities and thermal diffusivities are presented in Supplementary Figure S2. The total thermal conductivity $\left(\kappa_{\text {tot }}\right)$ is essentially the sum of the electronic $\left(\kappa_{\text {ele }}\right)$ and lattice $\left(\kappa_{\text {lat }}\right)$ contributions. Hence, $\kappa_{\text {lat }}$ can be estimated by subtracting $\kappa_{\text {ele }}$ from $\kappa_{\text {tot }}$. The electronic part $\left(\kappa_{\text {ele }}\right)$ is roughly proportional to the electrical conductivity $(\sigma)$ through the Wiedemann - Franz law, $\kappa_{\text {ele }}=L \sigma T$, where $L$ is the Lorenz number; here, $1.5 \times 10^{-8} \mathrm{~W} \Omega \mathrm{K}^{-2}$ (nondegenerate limit) was used. ${ }^{23}$ The $\kappa_{\text {ele }}$ is shown in Supplementary Figure S2c. For all nano-PbS samples, the thermal conductivity contribution from electrons was estimated to be $<0.35 \%$ of the $\kappa_{\text {tot }}$, indicating that phonons dominate thermal transport. In fact, the above calculation for $\kappa_{\text {lat }}$ is based on the assumption that only a single type of charge carrier is responsible for charge transport. However, thermoelectric materials are narrow-band semiconductors and always have both types of carriers (holes and electrons), especially at high temperatures. Minority carriers jump across the band gap, and the diffusing electron-hole pair gives rise to an additional thermal conductivity contribution, that is, bipolar thermal conductivity, that should be added to the total thermal conductivity. If this term is present, $\kappa_{\text {lat }}$ could tend to be overestimated. ${ }^{24}$ For ingot-PbS, the upturn above $723 \mathrm{~K}$ shown in Figures $3 \mathrm{a}$ and $\mathrm{b}$ is suggestive of the bipolar effect. Importantly, the bipolar effect is an obstacle to improving thermoelectric performance, as it increases thermal conductivity and also decreases the overall Seebeck coefficient through the excitation of minor carriers at high temperatures. In contrast, an upturn in the micro-PbS sample is not apparent at high temperatures because the grain size of micro-PbS was reduced to several hundred nanometers compared with the above $200 \mu \mathrm{m}$ grains of ingot- $\mathrm{PbS} .{ }^{23}$ When the grain size was reduced further to tens of nanometers (nano-PbS), the upturn is observed in the thermal conductivity curve at high temperatures, indicating a 'suppression' of the bipolar effect. ${ }^{6}$ Here, 'suppression' only refers to suppressing the contribution of the bipolar effect to the total thermal conductivity because the bipolar effect is still present (Figure $2 b$ ). The mechanism of this 'suppression' could be related to electron scattering by the high density of nanoscale grain boundaries. The upturn because of the bipolar effect in thermal conductivity is mainly related to charge carrier transport. In the present work, the electronic thermal conductivity of nano- $\mathrm{PbS}$ is quite small, $<8 \%$ (at $923 \mathrm{~K}$ ) of the total contribution. Thus, the bipolar effect is not obvious in thermal conductivity (Figures 3a and b), whereas an upturn in the Seebeck coefficient is observed at high temperatures because of its strong dependence on charge carrier transport (Figure $2 \mathrm{~b}$ ). To summarize, nano-PbS suppresses the electronic and bipolar contributions to the total thermal conductivity through electron scattering at high density of grain boundaries, although electrical properties are deteriorated to some degree.

Of course, the other important factor for reducing the total thermal conductivity is to lower the lattice thermal conductivity through strong phonon scattering at a high density of grain boundaries. Further analysis shows that both $\kappa_{\text {tot }}$ and $\kappa_{\text {lat }}$ of the three nano-PbS samples exhibit the same decreasing trend with increasing temperature over the entire temperature range tested. At room temperature, $\kappa_{\text {tot }} \approx \kappa_{\text {lat }} \approx 0.95,0.85$ and $0.88 \mathrm{~W} \mathrm{~m}^{-1} \mathrm{~K}^{-1}$ for nano-PbS- 1 , nano$\mathrm{PbS}-2$ and nano-PbS-3, respectively. These values are much lower than those of ingot-PbS $\left(\kappa_{\text {tot }} \approx 2.66, \kappa_{\text {lat }} \approx 2.53 \mathrm{Wm}^{-1} \mathrm{~K}^{-1}\right)$ and micro-PbS $\left(\kappa_{\text {tot }} \approx 2.41, \kappa_{\text {lat }} \approx 2.36 \mathrm{Wm}^{-1} \mathrm{~K}^{-1}\right)$. At $923 \mathrm{~K}$, an extremely low thermal conductivity $\left(\kappa_{\text {tot }} \approx 0.53, \kappa_{\text {lat }} \approx 0.5 \mathrm{Wm}^{-1} \mathrm{~K}^{-1}\right)$ can be obtained in nano- $\mathrm{PbS}$ samples that is the lowest value reported to date for PbQ-based $(\mathrm{Q}=\mathrm{Te}, \mathrm{Se}, \mathrm{S})$ thermoelectric materials. Notably, both the total and lattice thermal conductivities showed a significant decrease in nano- $\mathrm{PbS}$ samples and were $\sim 65 \%$ of that of the ingot sample. This decrease indicates that the lattice thermal conductivity was significantly reduced by the high density of grain boundaries. Because of this significant reduction in lattice thermal conductivity, theoretical calculations were carried out that will be discussed later.

By combining the significant reduction of lattice thermal conductivity and 'suppression' of the bipolar effect (electronic contribution) by strong phonon and electron scattering at a high density of grain boundaries, respectively, high thermoelectric performance can be achieved. Although the electrical properties were deteriorated to some degree, the reduction of thermal conductivity more than compensated for the reduction of the electrical properties. Using the electrical and thermal transport properties, the $Z T$ was calculated and is presented in Figure 3c. The maximum $Z T$ value at $923 \mathrm{~K}$ increased from 0.3 for ingot-PbS to 0.5 for nano-PbS-2, and showed no sign of saturation at the highest temperatures. This value is the highest in $\mathrm{PbQ}$ nanocrystalline materials and is also higher than the 

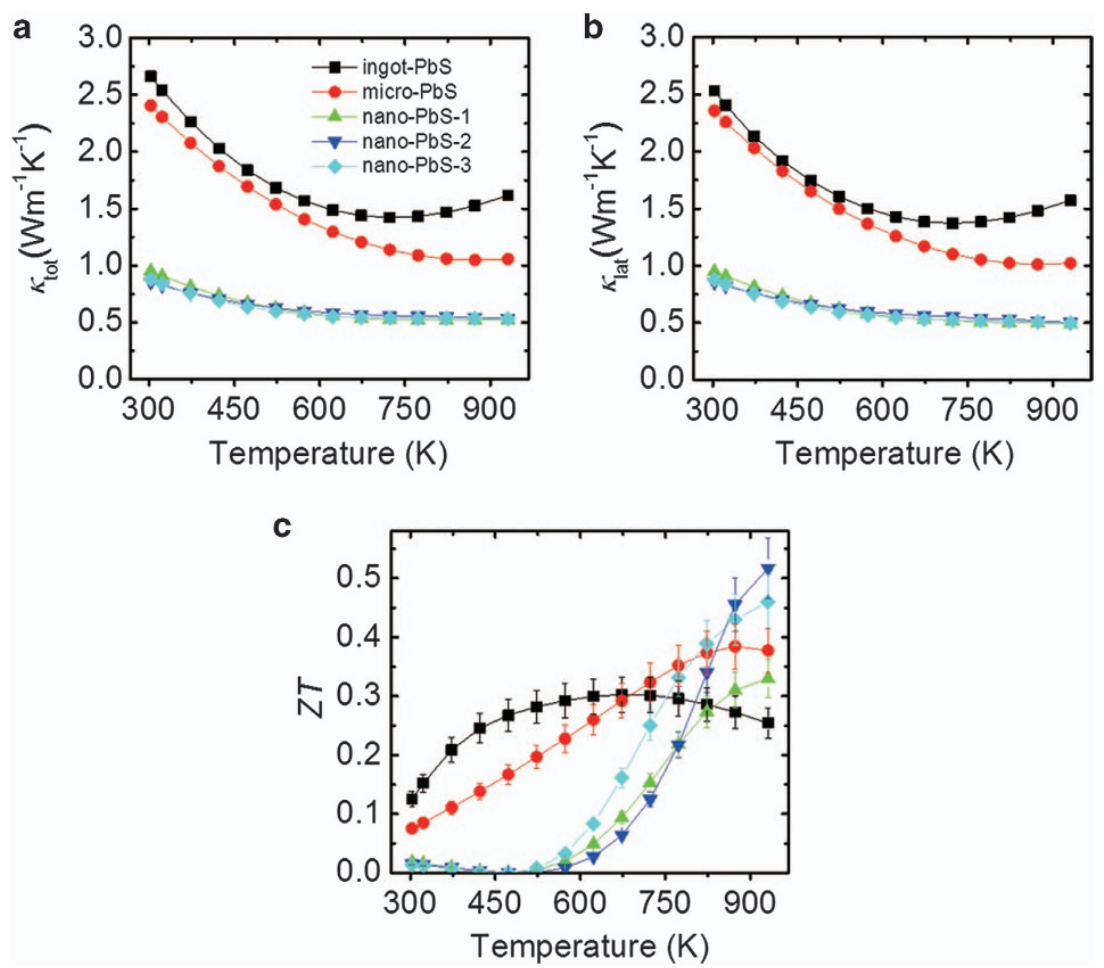

Figure 3 Thermoelectric properties as a function of temperature for PbS samples. (a) Total thermal conductivity. (b) Lattice thermal conductivity. (c) Figure of merit, $Z T$. The combined uncertainty for all measurements involved in the calculation of $Z T$ is $<15 \%$.

highest $Z T$ value of 0.35 reported for PbTe-PbSe core-shell nanocrystalline materials, ${ }^{17}$ as well as the $Z T=0.38$ at $670 \mathrm{~K}$ reported for nanocrystalline $\mathrm{Ag}_{2} \mathrm{Te}-\mathrm{PbTe}$ materials. ${ }^{25}$ The low average $\mathrm{ZT}$ for nano-PbS samples over the whole operational temperatures stems from the extremely low electrical conductivity at low temperatures. Efforts to enhance electrical transport in nanocrystalline $\mathrm{PbS}$ by subtly tuning the carrier concentration ${ }^{26}$ are ongoing. One may argue that the nanoscale grains in $\mathrm{PbS}$ are unstable at elevated temperatures. Usually, grain growth will occur when the small size (several $\mathrm{nm}$ ) grains are subjected to high temperatures. ${ }^{27}$ However, larger size (tens of $\mathrm{nm}$ or larger) grains should have enough resistance against the thermal ripening at high temperatures because the energy required for grain growth will dramatically increase as grain size increases. Therefore, not all nanomaterials are thermodynamically unstable ${ }^{28}$ and this should be the case for our nanocrystalline $\mathrm{PbS}$.

\section{Microstructural features}

To explore the microstructure of nano- $\mathrm{PbS}$ and investigate its relationship with thermoelectric performance, we employed various analytical TEM techniques to study the microstructural features of the nano-PbS-2 (Figures 4 and 5 and Supplementary Figures S3 and S4) and nano-PbS-3 (Figure 6 and Supplementary Figures S5 and S6) samples. For the nano-PbS-2 sample, the presence of nanoscale grains with sizes of $\sim 33 \mathrm{~nm}$ (or more specifically in the $14-58 \mathrm{~nm}$ interval) is evident in Figures 4a and b. The HRTEM image (Figure 4c) and its FFT image (Figure $4 \mathrm{~d}$ ) reveal low-angle grain boundaries. The grain boundary is a semi-coherent interface along the (002) plane. The relative rotation angle between two grains is $\sim 2.25^{\circ}$, so small that the spot splitting can only be observed at high-order reflections. Meanwhile, a high-angle grain boundary can also be found, as shown in Supplementary Figure S3. Because the grain size is very small, overlap between grains is also common in the sample. The high-magnification image (Figure $5 \mathrm{a}$ ) shows a relatively small grain $(\sim 15 \mathrm{~nm})$, marked with yellow dashed lines, located below a larger grain. Figure $5 \mathrm{~b}$ shows a representative HRTEM image of a homogeneous crystal lattice along the [001] zone axis. Although the majority of nanograins are free of nanoscale precipitates, small amounts of platelet-like precipitates $(\sim 1.3 \mathrm{~nm}$, ranging $0.5-2.2 \mathrm{~nm})$ can be observed inside some nanograins because of $\mathrm{Pb}$ enrichment (Figure $5 \mathrm{c}$ ). The projection traces of the nanoscale precipitates direct along [200] and [020], as shown in Figure 5c. Precipitate growth in a preferred direction reflects structural anisotropy. The small amount of $\mathrm{Pb}$-rich precipitates inside the nanograins can also act as phonon scattering centers to some extent.

Low-magnification annular bright-field $(\mathrm{ABF})$ and HAADF images are shown in Supplementary Figures S4 and S5, respectively. Along with EDS, these images can be used to compare the elemental concentrations in the precipitates and the matrix. Figure $5 \mathrm{~d}$ and the inset in Supplementary Figure S5 show the statistics results from EDS spot analysis of nano-PbS-2 and nano-PbS-3 samples, respectively. Although it is difficult to quantitatively determine the exact compositions of individual precipitates because of their overlap with the matrix, the EDS analysis still qualitatively indicates a large increase in the $\mathrm{Pb}$ signal for the precipitates compared with the matrix, suggesting that the precipitates are $\mathrm{Pb}$ rich. The $\mathrm{ABF}$ images (Supplementary Figure S5a) and HAADF (Supplementary Figure S5b) images from the same area clearly show the precipitates (they appear darker in the bright-field image and brighter in the dark-field image) with more high- $Z$ elements, consistent with the EDS spot analysis shown in the inset in Supplementary Figure S5. For nano-PbS-2, the concentration of $\mathrm{Pb}$ in the precipitates is $\sim 57.4 \%$, whereas it is $52.8 \%$ in the matrix (Figure $5 \mathrm{~d}$ ); for nano-PbS-3, it is $\sim 58 \%$ in the precipitates and $52.9 \%$ in the matrix (inset of Supplementary Figure S5). 

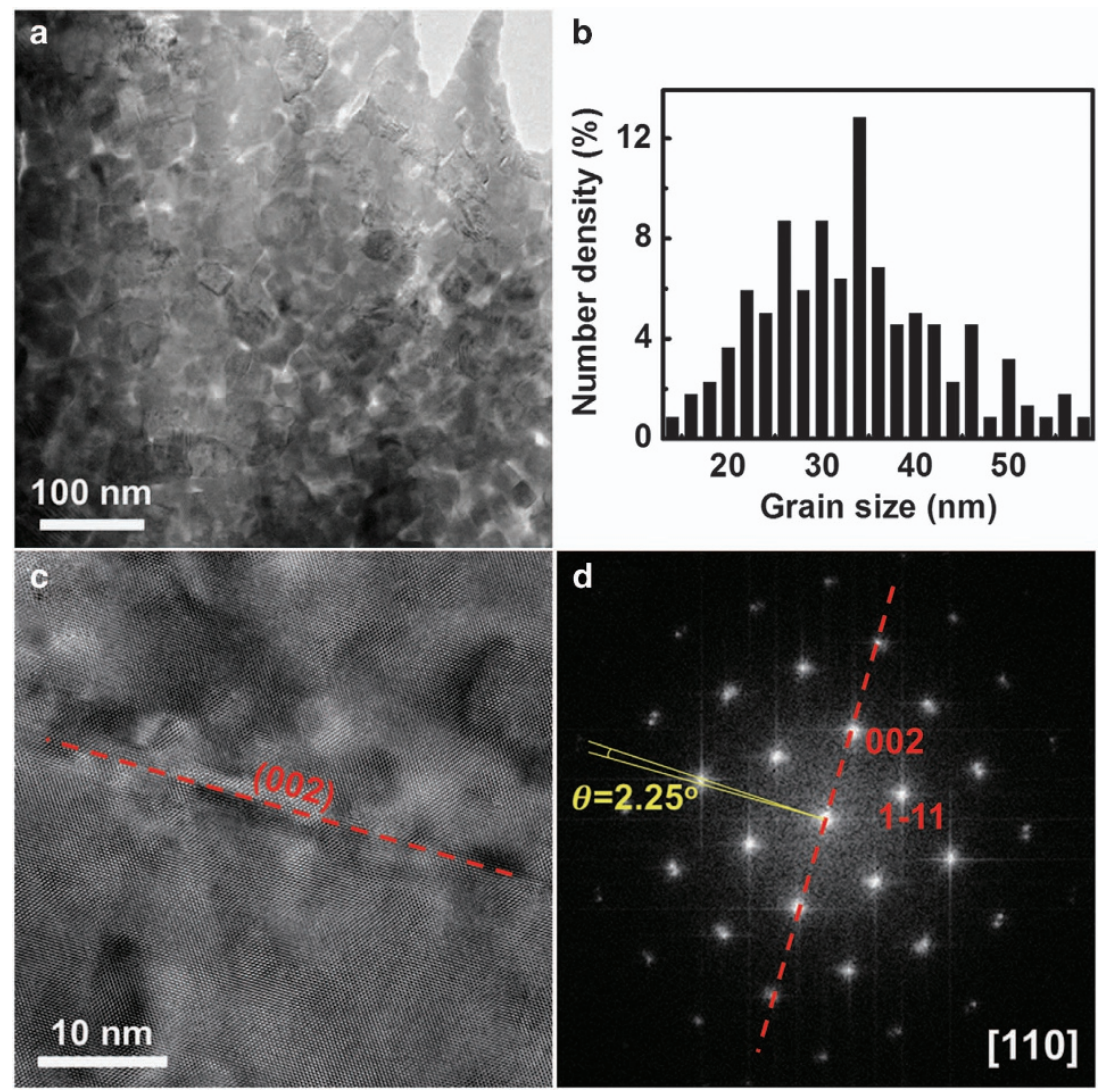

Figure 4 Microstructure of the nano-PbS-2 sample. (a) Low-magnification TEM image revealing nanoscale grains. (b) Grain size distribution histogram. (c) High-magnification lattice image showing a (002) grain boundary. (d) FFT image of (c) depicting the low-angle grain boundary along the [110] zone axis.

A higher density of precipitates can be observed in the nano-PbS-3 sample compared with the nano-PbS-2 sample because the former had a higher excess of $\mathrm{Pb}$ during preparation. These nanoscale precipitates reflect as platelet-like and spherical/ellipsoidal, with three crystallographic variants, as shown in Figure 6a and Supplementary Figures S5 and S6, respectively. The size of precipitates ranges from 0.7 to $3.6 \mathrm{~nm}$, and the average size is $\sim 1.4 \mathrm{~nm}$, as shown in the size distribution in Figure $6 \mathrm{~b}$. Figure $6 \mathrm{c}$ shows a representative HRTEM image of platelet-like precipitates obtained with the electron beam along the [001] zone axis; the inset SAED pattern confirms the endotaxially aligned structure between $\mathrm{Pb}$-rich precipitate and $\mathrm{PbS}$ matrix. The platelet-like precipitates are organized either perpendicular or parallel to each other. They can appear as single plates (marked with red dashed ellipsoidal boxes) as well as crossed plates (marked with yellow dashed rectangular boxes). A schematic of the crossed precipitate morphology in three-diensional space shows two parallel plates crossed with another plate, and the arrowhead indicates the direction of the TEM observations. To analyze the possible strain around the interface between the platelet-like precipitate and the matrix, high-quality HRTEM images were analyzed by geometric phase analysis, a semiquantitative lattice image-processing approach for revealing spatially distributed strain fields. ${ }^{29}$ Figure $6 \mathrm{~d}$ shows enlarged images and their analyzed geometric phase analysis results, namely the components $\varepsilon_{\mathrm{xx}}$ and $\varepsilon_{\mathrm{yy}}$ of the strain. The image shows two perpendicular platelet-like precipitates enclosed by dotted lines, and indicates that there is elastic strain only along the $x$ direction for the left precipitate and only along the $y$ direction for the right one. Thus, the strain distribution in platelet-like precipitates is anisotropic.
This result is in contrast to spherical precipitates that have more uniform omnidirectional strain distributions. ${ }^{5}$ The platelet-like precipitates with the plate plane along $\{200\}$ appear spherical/ ellipsoidal if we observe them from [110] or other non-[100] directions, ${ }^{9,29}$ as shown in Supplementary Figure S6. The PbS matrix and $\mathrm{Pb}$-rich nanosized precipitates have similar symmetry, structure and lattice parameters, and the corresponding crystallographic planes and directions are completely aligned in three dimensions. Thus, they can effectively scatter phonons without deteriorating charge transmission. $^{5}$

\section{Theoretical calculations}

Purely phenomenological models ${ }^{30}$ with a gray model assumption might be incapable of offering detailed insight into the mechanisms of thermal conduction and their interplay with the nanograins in nanocrystalline $\mathrm{PbS}$ materials. A strict treatment should involve detailed study of phonon propagation in the full phonon spectrum of the material. Today, $a b$ initio calculations are optimal sources of this information, as they avoid the need for experimental input as well as the transferability problems of fitting parameters. ${ }^{31-33}$ Thus, a theoretical model based on a combination of first-principles phonon calculations for pristine $\mathrm{PbS}$ and a set of semiempirical phonon scattering rates was constructed to understand the underlying mechanism and importance of grain boundary phonon scattering. A local density approximation, ${ }^{34}$ in conjunction with projectoraugmented-wave pseudopotentials, ${ }^{35}$ was employed for firstprinciples calculations, as implemented in the plane-wave-based density functional theory package VASP (on the basis of software 

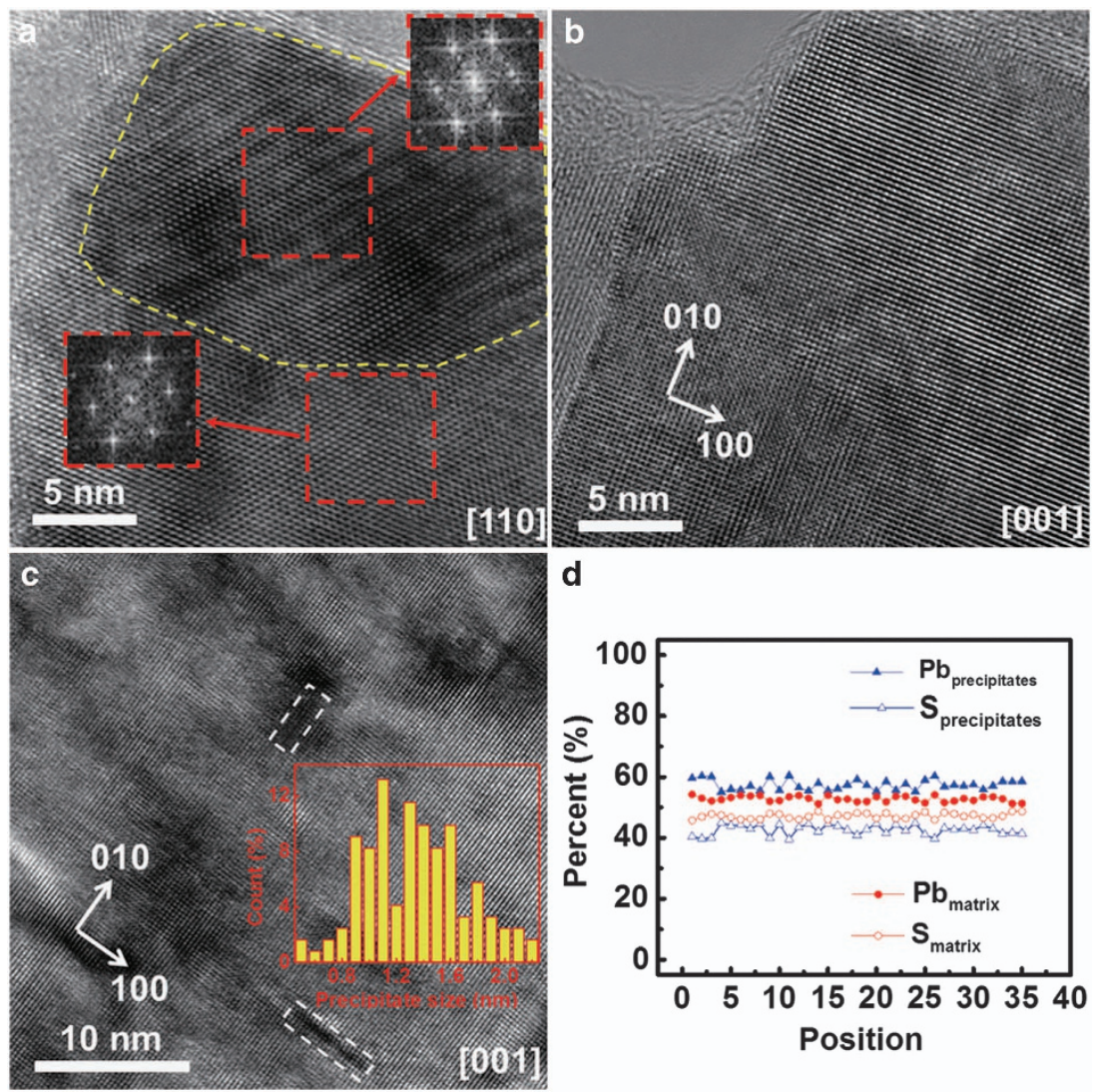

d

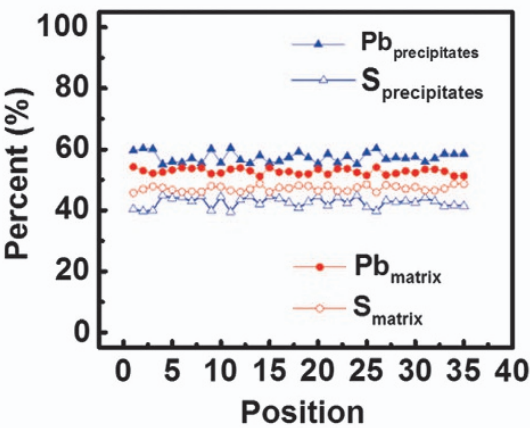

Figure 5 Microstructure of the nano-PbS-2 sample. (a) High-magnification lattice image along [110] zone axis showing the overlap between two grains. Insets are FFT images from the marked areas. (b) High-magnification lattice image showing an area without precipitates. (c) High-magnification lattice image showing a small density of platelet-like precipitates parallel or perpendicular to each other. The inset is the precipitate-size distribution histogram. (d) EDS elemental analysis of precipitates and the matrix.

licence agreements with the University of Vienna, Vienna, Austria). ${ }^{36}$ The energy cutoff was determined by adding $30 \%$ to the highest energy cutoff for the pseudopotentials. We checked the results of our structural minimizations by comparing our equilibrium lattice parameter of $0.585 \mathrm{~nm}$ with the experimental value of $0.593 \mathrm{~nm}$. The phonon dispersions obtained from our supercell calculations were also compared with previous theoretical calculations and with experimental data. ${ }^{37,38}$

In general, the lattice thermal conductivity can be obtained by summing the contribution of phonons from each branch $\alpha$ integrated over the Brillouin zone, as

$$
\kappa=\frac{h^{2}}{4 \pi^{2} k T^{2}} \sum_{\alpha} \int_{B Z} n_{0}\left(n_{0}+1\right)\left|v_{z}\right| \omega^{2} \Lambda d^{3} q,
$$

where $v_{z}$ is the phonon's group velocity in the transport direction, $n_{0}$ denotes the Bose-Einstein distribution and $\Lambda$ is the mean free path. For the bulk material, we incorporated impurity and anharmonic terms in their standard forms into the mean free path, ${ }^{39}$

$$
\Lambda_{\text {bulk }}^{-1}=A_{1} \omega^{4}+B_{1} \omega^{2} T \exp \left(-B_{2} / T\right) \text {. }
$$

The best-fit parameters obtained for the micro- $\mathrm{PbS}$ sample data for temperatures $<700 \mathrm{~K}$ (where the charge carrier contribution is negligible) were $A_{1}=3.37 \times 10^{-40} \mathrm{~s}^{4} \mathrm{~m}^{-1}, B_{1}=1.90 \times 10^{-19} \mathrm{~s}^{2} \mathrm{~m}^{-1}$ $\mathrm{K}^{-1}$ and $B_{2}=454.05 \mathrm{~K}$. The calculated result was in excellent agreement with our experimental data (see Figure 7), and further justifies this choice of functional form. However, in the nano- $\mathrm{PbS}$ samples, two additional terms, representing the contributions to phonon scattering from the nanograined structure and from the precipitates, have to be considered. These mechanisms operate at different length scales, and hence it is reasonable to incorporate them with Matthiesen's rule:

$$
\Lambda_{\text {nano }}^{-1}=\Lambda_{\text {bulk }}^{-1}+\Lambda_{\text {grain }}^{-1}+\Lambda_{\text {precipitates }}^{-1} .
$$

The construction of these terms is greatly facilitated by the availability of detailed experimental data. Potential barriers between grains impose a limitation to the phonon mean free path that is equal to the average reciprocal grain size times a form factor $F^{40}$ The effect of $\mathrm{Pb}$-rich precipitates is twofold: they act as localized scattering centers and, by changing the effective medium for phonons, they can affect the anharmonic term in $\Lambda_{\text {bulk }}^{-1}$. By treating the precipitates as approximately spherical particles of (variable) radius $R$ distributed randomly in the sample with density $\rho$, the first term can also be constructed from experimental data and a single adjustable dimensional factor $F_{A}{ }^{41}$ whereas the second term will be treated as a perturbation to the $B_{1}$ coefficient in Equation (5). Thus,

$$
\Lambda_{\text {precipitates }}^{-1}=F_{A} \frac{4}{9} \rho \pi\left\langle R^{2}\right\rangle\left(\frac{\langle R\rangle}{c}\right)^{4} \omega^{4}+B_{1} \delta B_{1} \omega^{2} T \exp \left(-B_{2} / T\right),
$$

where $c$ is the speed of sound in $\mathrm{PbS}^{30,42}$ The best-fit parameters found for the nano-PbS-2 sample were $F=0.279, F_{A}=1.09 \times 10^{3}$ and $\delta B=0.57$. Existing modeling efforts suggest that $F$ arises not only 


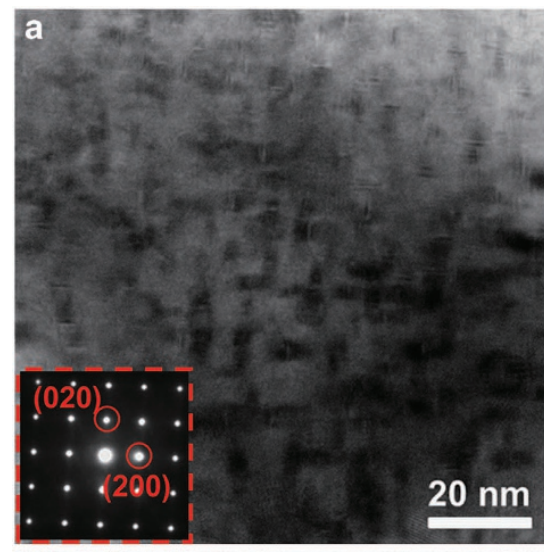

b
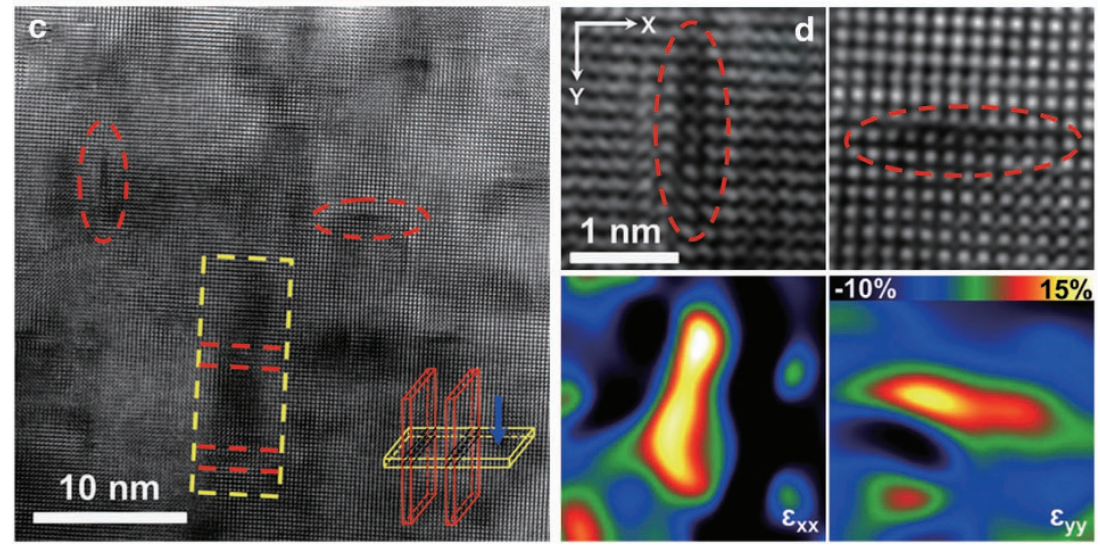

Figure 6 Microstructure of the nano-PbS-3 sample. (a) Medium-magnification TEM image showing the presence of platelet-like precipitates. (b) Histogram of precipitate-size distribution. (c) High-magnification lattice image showing many perpendicular or parallel platelet-like precipitates along 〈200〉 directions. The inset FFT image confirms the endotaxially aligned structure between the Pb-rich precipitate and PbS matrix. An inset schematic of the corresponding precipitate morphology in three-dimensional space represents two plate-like precipitates perpendicular to each other, and the arrowhead indicates the direction of the TEM observations. (d) Lattice image and strain maps showing elastic strain (color scale) along only one direction in the platelet-like precipitates.

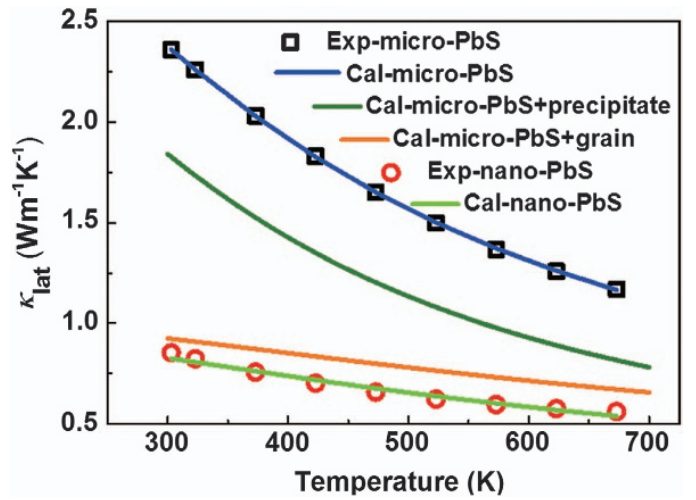

Figure 7 Fit of the theoretical model to the experimental thermal conductivity data of micro-PbS and nano-PbS-2. The separate effects of nano-grains and nano-precipitates are also shown.

from geometric features of the grains but also from how efficiently their boundaries can scatter phonons. ${ }^{43}$ In this context, an $F$ appreciably $<1.0$ can be interpreted plausibly as a sign of less than totally diffusive boundaries. $F_{\mathrm{A}}$ has been shown to be proportional to the square of the relative difference in densities between the host matrix and the precipitates. Because this difference is close to zero in the present study, scattering by precipitates is expected to be a comparatively minor factor that is confirmed in the following paragraphs.

Figure 7 shows a satisfactory fit of the above theoretical model to the experimental lattice thermal conductivity of micro-PbS and nano-PbS. More importantly, the separate effects of the terms $\Lambda_{\text {grain }}^{-1}$ and $\Lambda_{\text {precipitates }}^{-1}$ on the lattice thermal conductivity are highlighted. When only considering nano-precipitates, the calculated lattice thermal conductivity is $1.83 \mathrm{Wm}^{-1} \mathrm{~K}^{-1}$ at room temperature that is $\sim 78 \%$ of that of pure micro-PbS $\left(2.36 \mathrm{Wm}^{-1} \mathrm{~K}^{-1}\right)$. However, if only nano-grains are considered, the reduction is much more effective, down to $0.92 \mathrm{Wm}^{-1} \mathrm{~K}^{-1}$ ( $\sim 39 \%$ of that of pure micro$\mathrm{PbS})$. Although both of these effects cause an important reduction in the lattice thermal conductivity, they collectively indicate that most of the resulted enhancement comes from the nanograins. The effect of $\mathrm{Pb}$-rich precipitates is minor in a first approximation to the problem.

The fact that grain boundaries and precipitate/matrix interfaces cause a modification of the effective medium observed by phonons large enough to change the coefficient of the anharmonic term by almost $65 \%$ is remarkable. Our calculations also showed that both interfaces/boundaries can effectively scatter phonons; however, phonon scattering by grain boundaries is dominant. Thus, the model yields results consistent with expectations based on experimental fact: the nano- $\mathrm{PbS}-2$ sample has a low density of $\mathrm{Pb}$-rich precipitates but also a very small grain size, making grain boundary scattering dominant. 


\section{CONCLUDING REMARKS}

By combining experiments and theoretical calculations, we have presented a systematic study of the thermal conductivity of $\mathrm{PbS}$ materials with granular structures belonging to different length scales. We found that nanoscale grains can dramatically reduce the lattice thermal conductivity and partially suppress the bipolar effect. This scattering mechanism dominates over the effect of scattering by nanoprecipitates. A theoretical model that calculated the full phonon spectrum $a b$ initio further confirmed nanoscale grain scattering as the main factor influencing the reduced lattice thermal conductivity of the nanocrystalline $\mathrm{PbS}$ materials. These $\mathrm{PbS}$ nanocrystallines stand out as possible robust and low-cost alternatives to other high-performance thermoelectric materials.

\section{ASSOCIATED CONTENT}

Electronic Supplementary Information available: powder XRD patterns of nano-PbS and ingot-PbS (Supplementary Figure S1); heat capacity, thermal diffusivity and electronic thermal conductivity as a function of temperature for PbS (Supplementary Figure S2); supporting microstructure for $\mathrm{PbS}$-nano-2 and $\mathrm{PbS}$-nano-3 samples (Supplementary Figures S3-S6).

\section{CONFLICT OF INTEREST}

The authors declare no conflict of interest.

\section{ACKNOWLEDGEMENTS}

This contribution was supported primarily by the startup of the South University of Science and Technology of China, supported by the Shenzhen government, and the national 1000 plan for young scientists. This work was also partially supported by a grant-in-aid of ' 985 Project' from Xi'an Jiaotong University, the National Natural Science Foundation of China (Grant No 21201138 and 11204228), the National Basic Research Program of China (2012CB619402 and 2014CB644003) and the Fundamental Research Funds for the Central Universities.

Author contributions: JQH and LDZ designed the entire project. LDZ synthesized the bulk samples and conducted the thermoelectric experiments. HJW, XTS and JQH performed the TEM experiments. JC and ZW performed the theoretical calculations. ZYZ and YQQ synthesized the PbS nanoparticles, HJW, JC, ZW, LDZ and JQH analyzed the results and wrote the manuscript.

1 Kanatzidis, M. G. Nanostructured thermoelectrics: the new paradigm? Chem. Mater. 22, 648-659 (2010).

$2 \mathrm{He}$, J., Kanatzidis, M. G. \& Dravid, V. P. High performance bulk thermoelectrics via a panoscopic approach. Mater. Today 16, 166-176 (2013).

3 Hsu, K. F., Loo, S., Guo, F., Chen, W., Dyck, J. S., Uher, C., Hogan, T., Polychroniadis, E. K. \& Kanatzidis, M. G. Cubic AgPb $\mathrm{SbTe}_{2}+\mathrm{m}$ : bulk thermoelectric materials with high figure of merit. Science 303, 818-821 (2004).

4 Biswas, K., He, J., Zhang, Q., Wang, G., Uher, C., Dravid, V. P. \& Kanatzidis, M. G. Strained endotaxial nanostructures with high thermoelectric figure of merit. Nat. Chem. 3, 160-166 (2011)

5 Ikeda, T., Haviez, L., Li, Y. \& Snyder, G. J. Nanostructuring of thermoelectric $\mathrm{Mg}_{2} \mathrm{Si}$ via a nonequilibrium intermediate state. Small 8, 2350-2355 (2012).

6 Poudel, B., Hao, Q., Ma, Y., Lan, Y., Minnich, A., Yu, B., Yan, X., Wang, D., Muto, A., Vashaee, D., Chen, X., Liu, J., Dresselhaus, M. S., Chen, G. \& Ren, Z. High-thermoelectric performance of nanostructured bismuth antimony telluride bulk alloys. Science 320, 634-638 (2008).

7 Martin, J., Wang, L., Chen, L. \& Nolas, G. S. Enhanced Seebeck coefficient through energy-barrier scattering in PbTe nanocomposites. Phys. Rev. B 79, 115311 (2009).

8 Zhu, G. H., Lee, H., Lan, Y. C., Wang, X. W., Joshi, G., Wang, D. Z., Yang, J., Vashaee, D., Guilbert, H., Pillitteri, A., Dresselhaus, M. S., Chen, G. \& Ren, Z. F. Increased phonon scattering by nanograins and point defects in nanostructured silicon with a low concentration of germanium. Phys. Rev. Lett. 102, 196803 (2009).

9 Biswas, K., He, J., Blum, I. D., Wu, C.-I., Hogan, T. P., Seidman, D. N., Dravid, V. P. \& Kanatzidis, M. G. High-performance bulk thermoelectrics with all-scale hierarchical architectures. Nature 489, 414-418 (2012).

10 Zhang, Y., Wang, H., Kräemer, S., Shi, Y., Zhang, F., Snedaker, M., Ding, K. Moskovits, M., Snyder, G. J. \& Stucky, G. D. Surfactant-free synthesis of $\mathrm{Bi}_{2} \mathrm{Te}_{3}-\mathrm{Te}$ micro - nano heterostructure with enhanced thermoelectric figure of merit. ACS Nano 5, 3158-3165 (2011).

11 Ibáñez, M., Zamani, R., Gorsse, S., Fan, J., Ortega, S., Cadavid, D., Morante, J. R. Arbiol, J., Cabot, A. \& Core-Shell Nanoparticles, A. s. Building blocks for the bottomup production of functional nanocomposites: $\mathrm{PbTe}-\mathrm{PbS}$ thermoelectric properties. ACS Nano 7, 2573-2586 (2013).

12 Lan, Y., Poudel, B., Ma, Y., Wang, D., Dresselhaus, M. S., Chen, G. \& Ren, Z. Structure study of bulk nanograined thermoelectric bismuth antimony telluride. Nano Lett. 9 , 1419-1422 (2009).

13 Bux, S. K., Blair, R. G., Gogna, P. K., Lee, H., Chen, G., Dresselhaus, M. S., Kaner, R. B. \& Fleurial, J.-P. Nanostructured bulk silicon as an effective thermoelectric material. Adv. Funct. Mater. 19, 2445-2452 (2009).

14 Liu, W., Yan, X., Chen, G. \& Ren, Z. Recent advances in thermoelectric nanocomposites. Nano Energ. 1, 42-56 (2012).

15 Heremans, J. P., Thrush, C. M. \& Morelli, D. T. Thermopower enhancement in lead telluride nanostructures. Phys. Rev. B 70, 115334 (2004).

16 Quan, Z., Siu Loc, W., Lin, C., Luo, Z., Yang, K., Wang, Y., Wang, H., Wang, Z. \& Fang, J. Tilted face-centered-cubic supercrystals of PbS nanocubes. Nano Lett. 12, 4409-4413 (2012)

17 Scheele, M., Oeschler, N., Veremchuk, I. Peters, S-0., Littig, A., Kornowski, A Klinke, C. \& Weller, H. Thermoelectric properties of lead chalcogenide core-shell nanostructures. ACS Nano 5, 8541-8551 (2011).

18 Popescu, A., Woods, L. M., Martin, J. \& Nolas, G. S. Model of transport properties of thermoelectric nanocomposite materials. Phys. Rev. B 79, 205302 (2009).

19 Caillat, T., Borshchevsky, A. \& Fleurial, J.-P. Properties of single crystalline semiconducting $\mathrm{CoSb}_{3}$. J. Appl. Phys. 80, 4442-4449 (1996).

20 Taylor, W. E., Odell, N. H. \& Fan, H. Y. Grain boundary barriers in germanium. Phys. Rev. 88, 867-875 (1952)

21 Seto, J. Y. W. The electrical properties of polycrystalline silicon films. J. Appl. Phys. 46 5247-5254 (1975)

22 Androulakis, J., Peter, S. C., Li, H., Malliakas, C. D., Peters, J. A., Liu, Z., Wessels, B. W., Song, J.-H., Jin, H., Freeman, A. J. \& Kanatzidis, M. G. Dimensiona reduction: a design tool for new radiation detection materials. Adv. Mater. 23, 4163-4167 (2011).

23 Zhao, L.-D., Lo, S.-H., He, J., Li, H., Biswas, K., Androulakis, J., Wu, C.-I., Hogan, T. P., Chung, D.-Y., Dravid, V. P. \& Kanatzidis, M. G. High performance thermoelectrics from earth-abundant materials: enhanced figure of merit in $\mathrm{PbS}$ by second phase nanostructures. J. Am. Chem. Soc. 133, 20476-20487 (2011).

24 Zhao, L. D., Wu, H. J., Hao, S. Q., Wu, C. I., Zhou, X. Y., Biswas, K., He, J. Q. Hogan, T. P., Uher, C., Wolverton, C., Dravid, V. P. \& Kanatzidis, M. G. All-scale hierarchical thermoelectrics: MgTe in PbTe facilitates valence band convergence and suppresses bipolar thermal transport for high performance. Energ. Environ. Sci. 6, 3346-3355 (2013)

25 Cadavid, D., Ibáñez, M., Gorsse, S., López, A., Cirera, A., Morante, J. \& Cabot, A. Bottom-up processing of thermoelectric nanocomposites from colloidal nanocrystal building blocks: the case of $\mathrm{Ag}_{2} \mathrm{Te}-\mathrm{PbTe}$. J. Nanopart. Res. 14, 1-10 (2012).

26 Wang, H., Pei, Y., LaLonde, A. D. \& Snyder, G. J. Heavily doped p-type PbSe with high thermoelectric performance: an alternative for PbTe. Adv. Mater. 23, 1366-1370 (2011)

27 Song, X., Zhang, J., Yue, M., Li, E., Zeng, H., Lu, N., Zhou, M. \& Zuo, T. Technique for preparing ultrafine nanocrystalline bulk material of pure rare-earth metals. Adv. Mater. 18, 1210-1215 (2006)

28 Minnich, A. J., Dresselhaus, M. S., Ren, Z. F. \& Chen, G. Bulk nanostructured thermoelectric materials: current research and future prospects. Energ. Environ. Sci. 2 466-479 (2009).

29 Hÿtch, M. J., Snoeck, E. \& Kilaas, R. Quantitative measurement of displacement and strain fields from HREM micrographs. Ultramicroscopy 74, 131-146 (1998).

$30 \mathrm{He}$, J., Girard, S. N., Kanatzidis, M. G. \& Dravid, V. P. Microstructure-lattice thermal conductivity correlation in nanostructured $\mathrm{PbTe}_{0.7} \mathrm{~S}_{0.3}$ thermoelectric materials. Adv. Funct. Mater. 20, 764-772 (2010)

31 Qiu, B., Bao, H., Zhang, G., Wu, Y. \& Ruan, X. Molecular dynamics simulations of lattice thermal conductivity and spectral phonon mean free path of PbTe: bulk and nanostructures. Comp. Mater. Sci. 53, 278-285 (2012).

32 Tian, Z., Garg, J., Esfarjani, K., Shiga, T., Shiomi, J. \& Chen, G. Phonon conduction in $\mathrm{PbSe}, \mathrm{PbTe}$, and $\mathrm{PbTe}_{1-\mathrm{x}} \mathrm{Se}_{\mathrm{x}}$ from first-principles calculations. Phys. Rev. B 85, 184303 (2012)

33 Dames, C. \& Chen, G. in Thermoelectrics Handbook: Macro to Nano (ed. Rowe, D. M.) Ch. 421 (Taylor \& Francis, New York, 2006).

34 Mingo, N. Calculation of Si nanowire thermal conductivity using complete phonon dispersion relations. Phys. Rev. B 68, 113308 (2003).

35 Perdew, J. P. \& Zunger, A. Self-interaction correction to density-functional approximations for many-electron systems. Phys. Rev. B 23, 5048-5079 (1981).

36 Blöchl, P. E. Projector augmented-wave method. Phys. Rev. B 50, 17953-17979 (1994)

37 Kresse, G. \& Furthmüller, J. Efficient iterative schemes for ab initio total-energy calculations using a plane-wave basis set. Phys. Rev. B 54, 11169-11186 (1996).

38 Wakabayashi, I., Kobayashi, H., Nagasaki, H. \& Minomura, S. The effect of pressure on the lattice parameters part I. PbS and PbTe Part II. Gd, NiO, and $\alpha$-MnS. J. Phys. Soc. Jpn 25, 227-233 (1968).

39 Kilian, O., Allan, G. \& Wirtz, L. Near Kohn anomalies in the phonon dispersion relations of lead chalcogenides. Phys. Rev. B 80, 245208 (2009).

40 Takashiri, M., Tanaka, S., Hagino, H. \& Miyazaki, K. Combined effect of nanoscale grain size and porosity on lattice thermal conductivity of bismuth-telluride-based bulk alloys. J. Appl. Phys. 112, 084315 (2012). 
41 Mingo, N., Hauser, D., Kobayashi, N. P., Plissonnier, M. \& Shakouri, A. 'Nanoparticlein-alloy' approach to efficient thermoelectrics: silicides in SiGe. Nano Lett. 9, 711-715 (2009).

42 He, J., Zhao, L.-D., Zheng, J.-C., Doak, J. W., Wu, H., Wang, H.-Q., Lee, Y., Wolverton, C., Kanatzidis, M. G. \& Dravid, V. P. Role of sodium doping in lead chalcogenide thermoelectrics. J. Am. Chem. Soc. 135, 4624-4627 (2013).

43 Minnich, A. J., Lee, H., Wang, X. W., Joshi, G., Dresselhaus, M. S., Ren, Z. F., Chen, G. \& Vashaee, D. Modeling study of thermoelectric SiGe nanocomposites. Phys. Rev. B 80,155327 (2009). (c) (1) (5) (2) This work is licensed under a Creative Commons Attribution-NonCommercial-ShareAlike 3.0 Unported License. The images or other third party material in this article are included in the article's Creative Commons license, unless indicated otherwise in the credit line; if the material is not included under the Creative Commons license, users will need to obtain permission from the license holder to reproduce the material. To view a copy of this license, visit http://creativecommons.org/licenses/by-nc-sa/3.0/

Supplementary Information accompanies the paper on the NPG Asia Materials website (http://www.nature.com/am) 